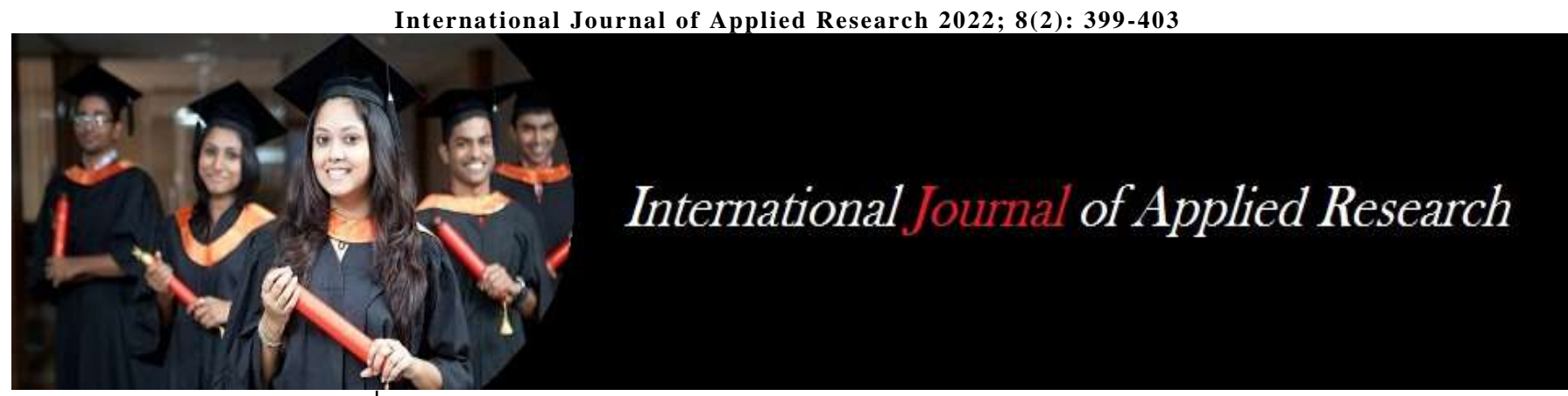

ISSN Print: 2394-7500 ISSN Online: 2394-5869 Impact Factor: 8.4 IJAR 2022; 8(2): 399-403 www.allresearchjournal.com Received: 08-12-2021 Accepted: 11-01-2022

Dr. Kishan Vyas PG Resident, Department of General Medicine, National Institute of Medical Science, Jaipur, Rajasthan, India

Dr. Eshan Sharma Professor, Department of General Medicine, National Institute of Medical Sciences, Jaipur, Rajasthan, India

Dr. Beauty Gupta Assistant Professor, Department of Pharmacy, MVGU, Jaipur, India

Corresponding Author: Dr. Kishan Vyas PG Resident, Department of General Medicine, National Institute of Medical Science, Jaipur, Rajasthan, India

\section{Serum ferritin level as a prognostic sign in cases of ischemic and hemorrhagic stroke: A prospective study}

\author{
Dr. Kishan Vyas, Dr. Eshan Sharma and Dr. Beauty Gupta
}

DOI: https://doi.org/10.22271/allresearch.2022.v8.i2f.9475

\section{Abstract}

Humans require iron to function. In cerebral ischemia, free radicals that utilize iron cause more damage. In this study, serum ferritin levels were looked at as a potential indicator of early neurologic worsening in patients with stroke. In this study, the maximum number of patients was in the 18-90year-old group. In total, 70 patients were admitted within 48 hours of a stroke. According to inclusion criteria, the study only included patients who were admitted within 48 hours of the onset of stroke. Of 70 patients, $34 \%$ were female and $65 \%$ were male.

A total of 32 patients with ischemic stroke were admitted, of which 25 improved and 7 deteriorated. Patients in the age group 61-70 were most likely to suffer an ischemic stroke. The mean serum ferritin level for improved patients was 211.61, while that of deteriorated patients was 316.82 .

In all, 38 patients with haemorrhagic stroke were admitted, out of which 29 improved and 9 deteriorated. Patients aged 61-70 were more likely to experience haemorrhagic strokes. A mean serum ferritin level of 228.67 was found in improved patients, whereas 364.27 was found in deteriorated patients.

The mean serum ferritin level in deteriorated patients is significantly higher than that of improved patients. Early neurologic deterioration is associated with high ferritin levels within 24 hours after stroke onset. In cerebral ischemia, increased body iron stores may enhance cytotoxic mechanisms contributing to stroke progression. More research is needed to identify the underlying causes and potential therapeutic implications of serum ferritin elevations. The reduction of serum ferritin levels can be achieved with medications like Deferoxamine.

Keywords: Ischemic stroke, hemorrhagic stroke, serum ferritin

\section{Introduction}

The World Health Organization defines stroke as a clinical syndrome characterized by a rapid onset of localized (or widespread with coma) neurological disruption lasting longer than 24 hours or causes death ${ }^{[1]}$.The diagnosis of stroke requires laboratory investigations including brain imaging. The number of deaths caused by cerebrovascular diseases in developed countries is rapidly rising as it is the third most common factor responsible for death following cardiovascular disease and cancer. Strokes are classified into ischemic strokes and hemorrhagic strokes.

1. The ischemic stroke occurs due to the blockage of a blood vessel which limits the supply of blood to the brain, whereas hemorrhagic stroke results from blood vessels ruptured in the brain which leads to blood spilling in the intracranial cavity.

2. Hemorrhagic strokes may be classified as intracerebral haemorrhages or subarachnoid haemorrhages depending on where blood spills Ischemic strokes account for $60-80 \%$ of all strokes. In both developed and developing countries, it contributes to morbidity and mortality.

The impact of this disease could be reduced by improved detection and modification of risk factors.

About $80 \%$ of stroke cases are caused by ischemic strokes, while $20 \%$ are caused by hemorrhagic strokes. However, the percentages of stroke types vary by population ${ }^{[2]}$. From the first Inter stroke study, 22 countries showed that stroke proportions African countries accounted for $66.7 \%$ and $34.8 \%$, accordingly, in comparison with $91.1 \%$ and $9.0 \%$. For developed nations, respectively ${ }^{[3]}$. 
Study data from the Stroke Investigations Research and Education Network (SIREN) study has reported a $68 \%$ ischaemic stroke rate and a $32 \%$ haemorrhagic stroke rate, in accordance with the prevalence of strokes among Africans in Inter stroke ${ }^{[4]}$. There is evidence of an evolution of stroke subtypes in few countries, including Ghana, resulting in a decline of hemorrhagic strokes while increasing the incidence of ischemic strokes ${ }^{[5,7]}$. Research done during the 1950s and 1980s in Ghana demonstrated that haemorrhagic stroke was the most prevalent form of stroke causing the majority of stroke deaths ${ }^{[5,6]}$.

\section{Materials and Methods}

This was a Single centred observational prospective hospital based study carried out in General medicine ward and ICU of NIMS Hospital Jaipur for a duration of 18 months (January 2020 to June 2021). Patients of stroke who were admitted within $48 \mathrm{hrs}$ of symptoms onset were included in the study and the diagnosis of stroke was confirmed by CT/MRI Brain. Neurological assessment was done by Canadian stroke scale. Serum ferritin levels was performed within $48 \mathrm{hrs}$ of onset of symptoms. Neurological assessment was repeated on 6th day of admission by Canadian stroke scale and patients were classified into 3 categories namely clinical improvement, deterioration and death. following data were recorded for each patient of acute stroke-NCCT/MRI Brain, Serum ferritin, Age, sex, history of Diabetes, Hypertension and smoking Biochemical profile including: Haemoglobin levels, blood sugar levels(random), liver function tests, renal function tests, lipid profile, serum electrolytes. All the information were collected on a pre-designed Performa. Data thus collected were entered in MS Excel 2007 worksheet in the form of master chart. These data were classified and analysed as per aims and objectives of the study. Inferences were drawn with use of appropriate test of significance.

\section{Results}

A total of 70 patients were enrolled in the study after an informed consent, out of which 46 were males and 24 were females with a age range of 20-90. 32patients were diagnosed with ischemic stroke and 38 with hemorrhagic stroke in which percentage of female was $34.38 \%$ and $34.21 \%$ respectively. Maximum no. of patients i.e; 23 were reported with the age range of $61-70,17$ patients with the age group 51-60,16 patients were from the aged range from 71-80 and 10 patients were from the age group 81-90.

The mean serum ferritin level of deteriorated patients was significantly higher as compared to those who improved in both cases i.e; 316.8271 and 364.2767 respectively. Mean statistically different with $p<0.001$.

\section{Patient Profile}

Total sample size: 70. Males: 46.

Females: 24.

Age range: 20-90.

\section{Demographic Details}

Followings were the findings of the present study.

\section{Patients distributed by etiological factor}

Table 1: Patients distributed by etiological factor

\begin{tabular}{|c|c|c|}
\hline Stroke & Nos. of Patients & Percentage \\
\hline Ischemic stroke & 32 & $45.71 \%$ \\
\hline Haemorrahgic stroke & 38 & $54.29 \%$ \\
\hline
\end{tabular}

Number of cases classified by type and results

Table 2: Distribution based on type and results

\begin{tabular}{|c|c|c|}
\hline Nos. of cases & Ischemic stroke & Hemorrhagic stroke \\
\hline Improved cases & 25 & 29 \\
\hline Deteriorated cases & 7 & 9 \\
\hline Total & 32 & 38 \\
\hline
\end{tabular}

Patients with ischemic stroke distributed on the basis of sex

Table 3: Sex distribution of patients with ischemic stroke

\begin{tabular}{|c|c|c|}
\hline Sex & Count of Genders & Percentage \\
\hline Male & 21 & $65.62 \%$ \\
\hline Female & 11 & $34.38 \%$ \\
\hline
\end{tabular}

Patients with hemorrhagic stroke distributed by sex

Table 4: Sex distribution of patients of haemorrhagic stroke

\begin{tabular}{|c|c|c|}
\hline Sex & Count of genders & Percentage \\
\hline Men & 25 & $65.79 \%$ \\
\hline Women & 13 & $34.21 \%$ \\
\hline
\end{tabular}

Distribution by age of stroke patients

Table 5: Distribution by age of stroke patients

\begin{tabular}{|c|c|c|}
\hline Age quartile & Ischemic stroke & Haemorrahgic stroke \\
\hline $21-30$ & 01 & 00 \\
\hline $31-40$ & 00 & 01 \\
\hline $41-50$ & 02 & 00 \\
\hline $51-60$ & 10 & 07 \\
\hline $61-70$ & 12 & 11 \\
\hline $71-80$ & 08 & 08 \\
\hline $81-90$ & 05 & 05 \\
\hline
\end{tabular}

Age-based mean of patients with ischemic stroke on the basis of prognosis

Table 6: Age-based mean of patients with ischemic stroke on the basis of prognosis

\begin{tabular}{|c|c|}
\hline Patient's Condition & Ischemic Stroke \\
\hline Patients mean age who improved & 69.88 \\
\hline Patients mean age who deteriorated & 67.00 \\
\hline
\end{tabular}

Age-based mean of patients with hemorrhagic stroke on the basis of prognosis

Table 7: Age-based mean of patients with haemorrhagic stroke on the basis of prognosis

\begin{tabular}{|c|c|}
\hline Patient's condition & Hemorrhagic Stroke \\
\hline Patients mean age who improved & 67.06897 \\
\hline Patients mean age who deteriorated & 64.44444 \\
\hline
\end{tabular}

\section{Mean serum ferritin in ischemic stroke patients}

Table 8: Mean serum ferritin of patients of ischemic stroke

\begin{tabular}{|c|c|}
\hline Patients Condition & Mean of serum ferritin \\
\hline Improved subject & 211.6124 \\
\hline Deteriorated subject & 316.8271 \\
\hline
\end{tabular}

Mean of serum ferritin in hemorrhagic stroke cases

Table 9: Mean serum ferritin of patients of haemorrhagic stroke

\begin{tabular}{|c|c|}
\hline Patients Condition & Mean of serum ferritin \\
\hline Improved subjects & 228.6731 \\
\hline Deteriorated subjects & 364.2767 \\
\hline
\end{tabular}




\section{Inference}

- In both groups, the means are statistically different with $p<0.001$.

- Ferritin levels are significantly higher in patients who deteriorate than those who improve.
T-test assuming disperse variances for comparison of mean serum ferritin in hemorrhagic stroke assuming unequal variances

Table 10: T-Test assuming unequal variances to compare mean of serum ferritin of improved and deteriorated groups in ischemic and hemorrhagic stroke

\begin{tabular}{|c|c|c|}
\hline & Improved & Deteriorated \\
\hline Mean & 228.6731 & 364.2767 \\
\hline Variance & 8513.476 & 8283.64 \\
\hline Observation & 29 & 9 \\
\hline Hypothesized mean difference & \multicolumn{2}{|c|}{135.6036} \\
\hline Df & \multicolumn{2}{|c|}{-7.7839} \\
\hline T stat & \multicolumn{2}{|c|}{$9.4 \mathrm{E}-07$} \\
\hline P(T<=t)one tail & \multicolumn{2}{|c|}{1.76131} \\
\hline T critical one tail & $1.88 \mathrm{E}-06$ \\
\hline P(T<=t)two-tail & \multicolumn{2}{|c|}{2.144787} \\
\hline T critical two-tail &
\end{tabular}

\section{Inference}

- In both groups, the means are statistically different with $p<0.001$.
- Ferritin levels are significantly higher in patients who deteriorate than those who improve.

\section{Patient distribution based on etiology}

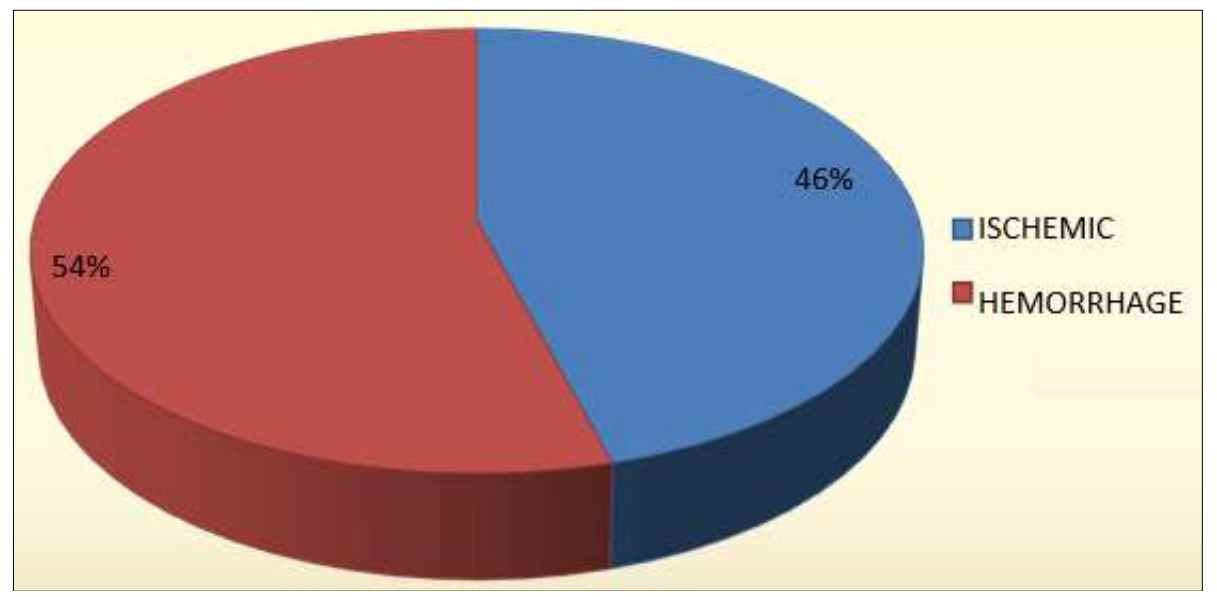

Fig 1: Patient distribution based on etiology

Patient's distribution on the basis of classification and results diagrammatic presentation in a bar graph

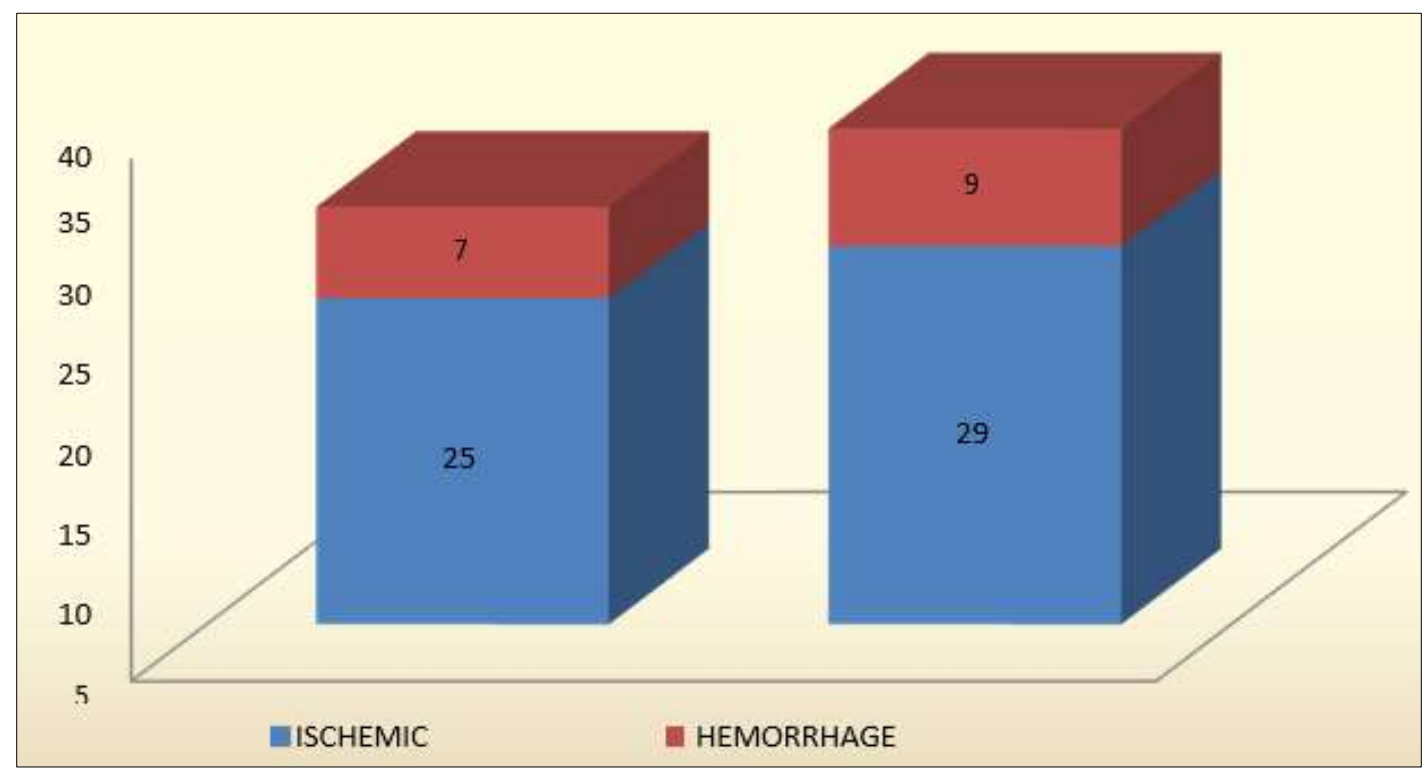

Fig 2: Graphical presentation based on type of stroke and outcome

$$
\sim 401 \sim
$$




\section{Graphical representation of sex distribution of cases}

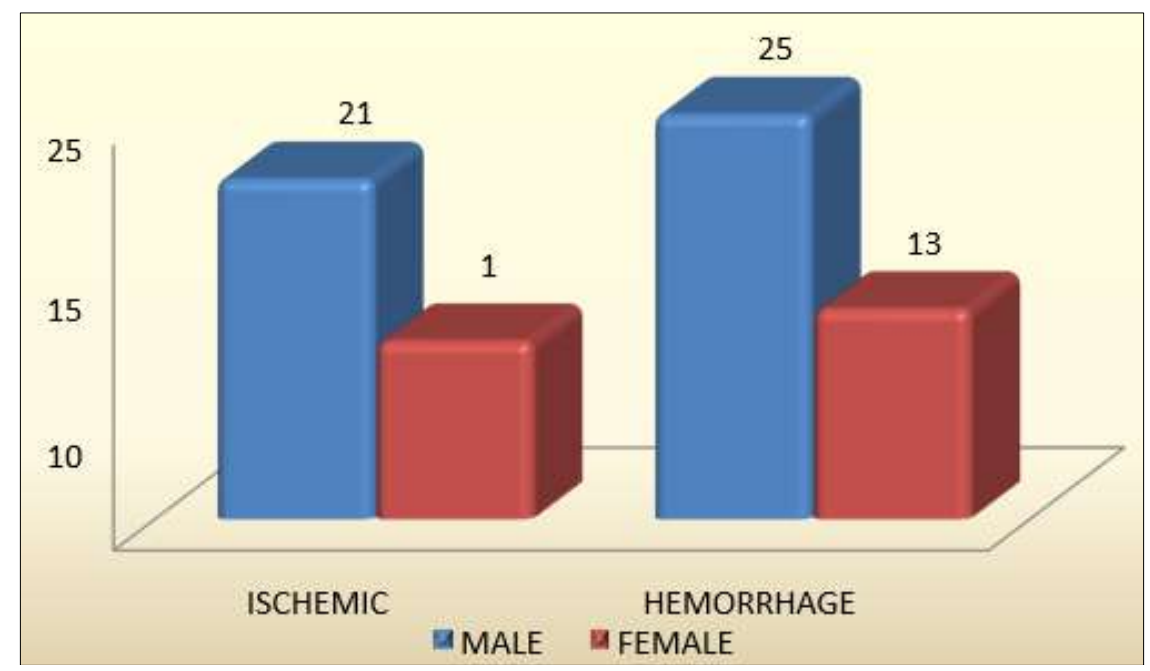

Fig 3: Graphical presentation of patients on the basis of gender determination

On the basis of progression in individuals with ischemic and hemorrhagic stroke: mean of age

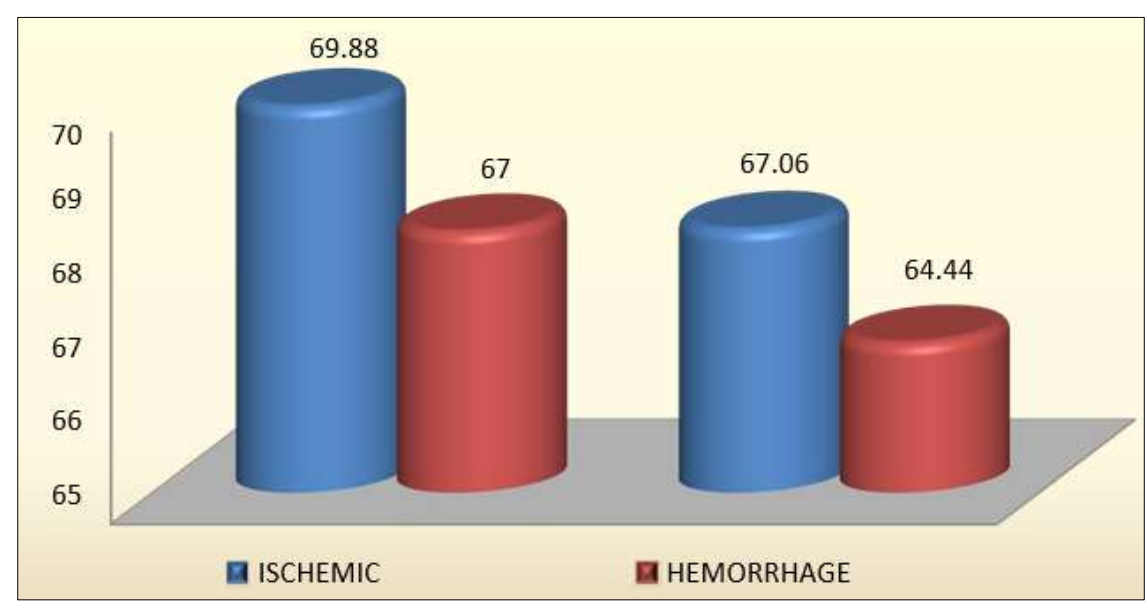

Fig 4: Mean age of patients of ischemic and haemorrhagic stroke based on prognosis

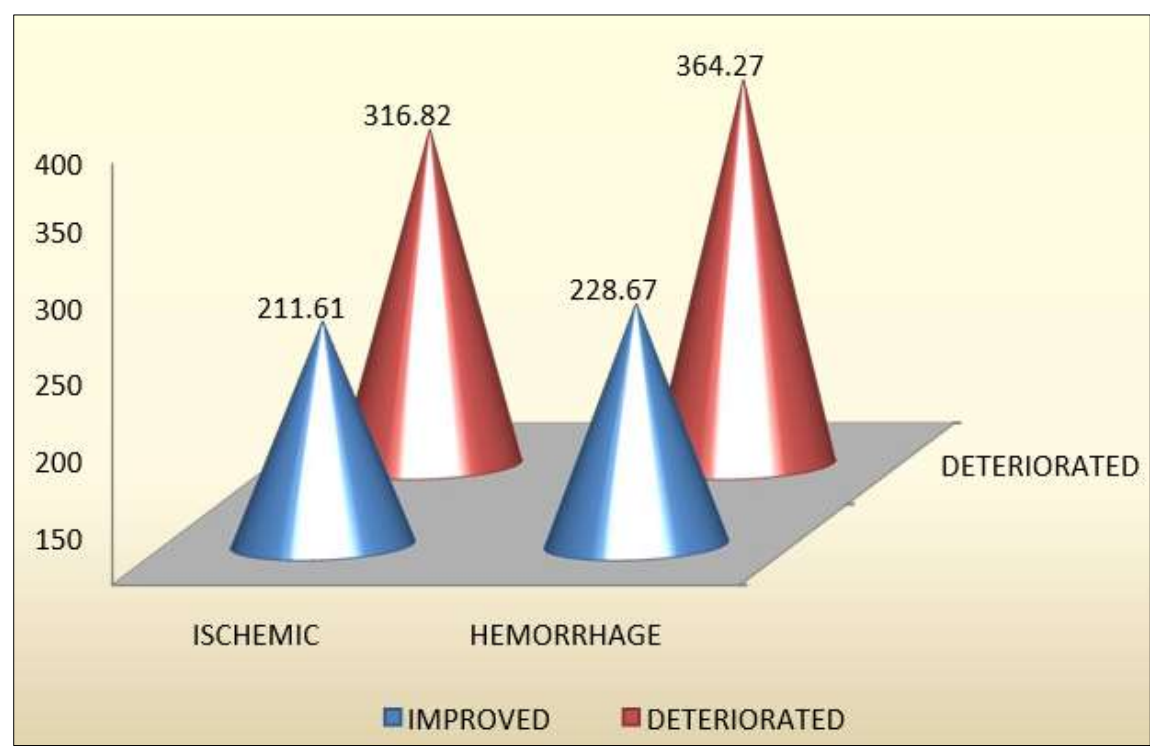

Fig 5: Graphical representation of mean serum ferritin level of patients of stroke based on prognosis 


\section{Discussion}

Study results suggest that ferritin levels could be an influential predictive criterion for strokes. There is a relationship between elevated ferritin concentration and neurodegeneration as a result of stroke. Serum ferritin measurements can thus be used to determine whether an individual is susceptible to stroke. Among the two groups with better and worsening health, the average age of the individuals was quite similar. Neither improved nor deteriorating groups differed in the prevalence of various other influences. Both the improving and deteriorating groups, nevertheless, ferritin levels were lower in the deteriorating group. It makes no difference if a stroke is Haemorrahgic or ischemic, that is true. Individuals who rapidly declined showed elevated ferritin values at the time of admission. Serum ferritin provides an indication of cellular ferritin concentrations, enabling us to discern whether infarcted tissues possess adequate iron stores ${ }^{[8,9]}$. Studies have shown elevated serum ferritin levels as well as high cerebrospinal fluid ferritin levels contributed to worsening neurological symptoms, negative outcomes, increased infarct size, and glutamate accumulation among stroke victims $[10,11]$. As a whole, the findings indicate excessive iron intake is responsible for:

1. Stroke patients experience negative outcomes neurologically.

2. Thrombolytic treatment interferes with iron overload.

3. Chlorination therapy is effective after a stroke when serum ferritin levels are high.

\section{Conclusion}

Our study has shown that increased serum ferritin levels predict stroke outcome. Serum ferritin levels above normal suggest enhanced medical protocols, indicating cognitive decline. Serum ferritin levels also aid in deciding whether to use thrombolytic therapy. There are ways to figure out whether a patient would benefit from thrombolysis. Patients with elevated serum ferritin are more likely to deteriorate following thrombolysis.

\section{References}

1. Wade S, Smith S, Claiborne Johnston, J Claude Hemphill III. Harrison's Principles of Internal Medicine. 19th Ed. 2, 2559-2586.

2. Warlow C P. Epidemiology of stroke. The Lancet. 1998;352(3):1-4.

Sacco RL, Kasner SE, Broderick JP, et al. An updated definition of stroke for the 21st century: a statement for healthcare professionals from the American heart association/American stroke association Stroke. 2013;44(7):2064-2089.

3. Adogu POU, Ubajaka CF, Emelumadu OF, Alutu COC. Epidemiologic Transition of Diseases and HealthRelated Events in Developing Countries: A Review. American Journal of Medicine and Medical Sciences. 2015;5(4):150-157.

4. Caplan LR Caplan's Stroke: A Clinical Approach. 3rd. Woburn, England. 2000.

5. Nilsen MLA. historical account of stroke and the evolution of nursing care for stroke patients. Journal of Neuroscience Nursing. 2010;42(1):19-27.

6. Ashrafian H. Familial stroke 2700 years ago. Stroke. 2010;41(4):187.
7. Walters GO, Miller FM, Worwood M. Serum ferritin concentrations and iron stores in normal subjects. J ClinPathol. 1973;26:770-772.

8. Connor JR, Menzies SL, St Martin SM, Mufson EJ. Cellular distribution of transferrin, ferritin, and iron in normal and aged human brains. J Neurosci Res. 1990;27:595-611.

9. Davalos A, Fernandez-Real JM, Ricart W, Soler S, Molins A, Planas E, Genis D. Iron-related brain damage in acute ischemic stroke. Stroke. 1994;25:1543-1546.

10. Davalos A, Castillo J, Marrugat J, Fernandez-Real JM, Armengou A, Cacabelos P. Rama R. Body iron stores and early neurological deterioration in acute cerebral infarction. Neurology. 2000;54:1568-1574. 